\title{
Dynamically Emerging Topological Phase Transitions in Nonlinear Interacting Soliton Lattices
}

\author{
Domenico Bongiovanni, ${ }^{1,2}$ Dario Jukić ${ }^{3}$ Zhichan Hu, ${ }^{1}$ Frane Lunić, ${ }^{4}$ Yi Hu, ${ }^{1}$ \\ Daohong Song, ${ }^{1}$ Roberto Morandotti, ${ }^{2,5}$ Zhigang Chen, ${ }^{1,6, \text { f }}$ and Hrvoje Buljan ${ }^{1,4, \dagger}$ \\ ${ }^{1}$ The MOE Key Laboratory of Weak-Light Nonlinear Photonics, \\ TEDA Applied Physics Institute and School of Physics, Nankai University, Tianjin 300457, China \\ ${ }^{2}$ INRS-EMT, 1650 Blvd. Lionel-Boulet, Varennes, Quebec J3X 1S2, Canada \\ ${ }^{3}$ Faculty of Civil Engineering, University of Zagreb, A. Kačića Miošića 26, 10000 Zagreb, Croatia \\ ${ }^{4}$ Department of Physics, Faculty of Science, University of Zagreb, Bijenička c. 32, 10000 Zagreb, Croatia \\ ${ }^{5}$ Institute of Fundamental and Frontier Sciences, \\ University of Electronic Science and Technology of China, Chengdu, Sichuan 610054, China \\ ${ }^{6}$ Department of Physics and Astronomy, San Francisco State University, San Francisco, California 94132, USA
}

(Dated: March 16, 2021)

\begin{abstract}
We demonstrate dynamical topological phase transitions in evolving Su-Schrieffer-Heeger (SSH) lattices made of interacting soliton arrays, which are entirely driven by nonlinearity and thereby exemplify emergent nonlinear topological phenomena. The phase transitions occur from topologically trivial-to-nontrivial phase in periodic succession with crossovers from topologically nontrivial-totrivial regime. The signature of phase transition is gap-closing and re-opening point, where two extended states are pulled from the bands into the gap to become localized topological edge states. Crossovers occur via decoupling of the edge states from the bulk of the lattice.
\end{abstract}

PACS numbers: 03.65.Vf, 42.65.Tg

Topological photonics offers a unique path for manufacturing photonic devices immune to scattering losses and disorder 1, 2. Since the pioneering theoretical predictions [3] and experimental demonstrations [4 of topologically protected electromagnetic edge states, most studies have focused on linear topological photonic structures 1, 2, However, by combining topology with nonlinearity [5-20], many opportunities for fundamental discoveries and new functionalities of the devices arise [21]; this is appealing also because nonlinearity inherently exists or is straightforwardly activated in most of the currently used linear topological photonic systems. The studies of nonlinear topological phenomena in photonics include, for example, nonlinear topological edge states and solitons [5] 13, 13, 18, topological phase transitions activated via nonlinearity 9 12 , nonlinear frequency conversion [19, 20, topological lasing [22 27], and nonlinear tuning of non-Hermitian topological states [28, 29].

In a recent study, we have introduced the concepts of inherited and emergent nonlinear topological phenomena [16. In this classification, inherited phenomena occur when nonlinearity is a small perturbation on an otherwise linear topological system. For example, in the SSH lattice [30, nonlinearity can easily break the chiral symmetry and therefore the underlying topology; this enables coupling into an otherwise topologically protected edge state [16, 17. However, many of the system properties, such as the structure of the nonlinear topological edge states and/or solitons [5, 7, 13, 18, are inherited from the corresponding linear system [16. In contradistinction, emergent nonlinear topological phenomena occur when the underlying linear system is not topological, but the nonlinearity induces nontrivial topology [16. Nonlinearity induced topological phase transitions 9.12 are examples of emergent nonlinear topological phenomena. In a recent experiment utilizing a nonlinear waveguide lattice structure [11], such a transition was shown to happen when power exceeded a certain threshold value. Emergent nonlinear topological phenomena are intriguing but were scarcely explored in nonlinear topological photonics.

Here we report the dynamical topological phase transitions entirely driven by nonlinearity, which constitute an example of emergent nonlinear topological phenomena. These phase transitions occur in colliding soliton lattices and are enabled by elastic soliton collisions. In optics, spatial solitons are stable localized optical beams, which occur when diffraction is balanced by nonlinearity 31 . Here we create two one-dimensional (1D) soliton sublattices, and initially kick them in opposite directions. As the sublattices evolve and collide, their superposition forms a paradigmatic model of topological physics: the SSH lattice [30, which can be in the topologically nontrivial [Fig. 11(a)] as well as trivial [Fig. 11(b)] phase featuring the so-called Zak phase [32. We find two kinds of interesting phenomena, which periodically occur in succession: (i) a dynamical topological phase transition from topologically trivial-to-nontrivial phase, characterized by a gap closing and re-opening at a single point, where two extended states are pulled from the bands into the gap to become localized topological edge states [see Fig. 1(c)]; (ii) a crossover from the topologically nontrivial-to-trivial regime, which occurs via decoupling of the edge states from the bulk of the lattice [see Fig. 11(d)].

We emphasize up front that there is a distinction be- 
tween our system, and those from Refs. 9 11, which all exhibit nonlinearity-induced topological phase transitions. In the theoretical models of Refs. [9, 10, the photonic lattices are fixed in $x$-space. In Ref. [1] they are fixed in the $x$-z-space (i.e., "spacetime"); the power of an external excitation can change the coupling via nonlinearity to induce a phase transition. In our system, the whole lattice autonomously nonlinearly evolves in the $x$ - $z$-space, resulting in different topological phases along $z$ (i.e., "time"). The surprising connection between interacting soliton lattices and nontrivial topology is revealed by the phase transitions and crossovers accompanied by the "birth" and "death" of topological edge states. This reminds of the surprising connection between topology and quasicrystals, also revealed by the phase transitions 33 .

We first outline a few basic facts about the SSH lattice. It is a $1 \mathrm{D}$ topological system, which exists due to the underlying chiral symmetry [2, 30]. In its topologically nontrivial phase, the intercell coupling $t^{\prime}$ is stronger than the intracell coupling $t\left(t<t^{\prime}\right)$ [see Fig. 11(a)]. The nontrivial SSH lattice has two topological edge modes with propagation constants residing in the band gap and a characteristic phase structure [30, 34]. In the trivial phase $t>t^{\prime}$ [see Fig. 1 (b)], there are two bands separated by a gap, and all eigenmodes are extended. This model has been implemented in versatile systems, including photonics and nanophotonics 34 38, plasmonics [39, 40, as well as quantum optics 41 44]. Some of the aforementioned nonlinear topological phenomena have been studied also in the nonlinear SSH model [810, 14, 17, 19, 26, 27.

We consider the propagation of a linearly-polarized optical beam in a nonlinear medium, which is described by a nonlinear Schrödinger equation (NLSE),

$$
i \frac{\partial \psi}{\partial z}+\frac{1}{2 k} \frac{\partial^{2} \psi}{\partial x^{2}}+\gamma|\psi|^{2} \psi(x, z)=0,
$$

where $\psi(x, z)$ refers to the electric field envelope, $\gamma$ defines the strength of the nonlinearity (we assume a Kerr-type nonlinearity), and $k$ is the wavenumber in the medium. The NLSE possesses a family of soliton solutions, with the hyperbolic-secant soliton being the most representative [45]:

$$
\begin{array}{r}
\psi_{S}(x, z ; \kappa, \theta)=\sqrt{I_{0}} \operatorname{sech}\left(\frac{x}{x_{0}}-\frac{\kappa z}{k x_{0}^{2}}\right) \\
\times \exp \left[i\left(\frac{\kappa}{x_{0}} x+\frac{1-\kappa^{2}}{2 k x_{0}^{2}} z+\theta\right)\right] .
\end{array}
$$

Here, $x_{0}$ is a scaling factor, $\kappa / x_{0}$ is the initial momentum, $I_{0}$ defines the peak intensity, and $\theta$ is an arbitrary phase. The stationary propagation is achieved when diffraction (quantified by the diffraction length $k x_{0}^{2}$ ) is balanced by the nonlinearity (quantified by the nonlinear length $\left.1 / \gamma I_{0}\right)$, that is, when $\gamma I_{0}=\left(k x_{0}^{2}\right)^{-1}$.

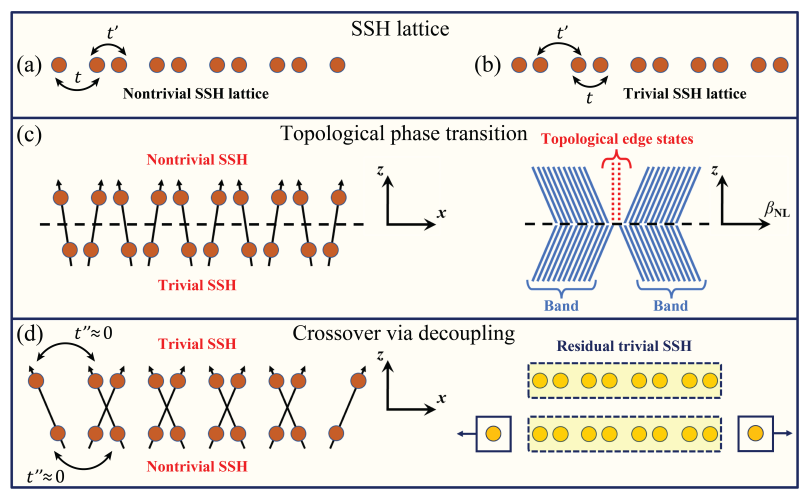

FIG. 1: Illustration of topological phase transition and crossover found in the evolving SSH soliton lattice. (a) The SSH lattice in the topologically nontrivial regime with $t<t^{\prime}$, characterized by two localized topological edge states. (b) The SSH lattice in the topologically trivial regime with $t>t^{\prime}$. (c) Sketch of the topological phase transition from trivialto-nontrivial phase in real space (left) and in the spectrum (right). At the phase transition, the gap closes, and two extended eigenmodes are pulled from the bands into the gap to become topological edge states. (d) Sketch of the crossover from the nontrivial-to-trivial phase via decoupling of the outermost lattice sites. The next-nearest-neighbor coupling is negligible in our SSH lattice, $t^{\prime \prime} \approx 0$, which results in decoupling during evolution in our system (left). This is equivalent to pulling off the outermost SSH lattice sites to infinity, leaving the residual lattice in the trivial phase (right).

Systems described by Eq. (1) are usually unrelated to nontrivial topology. Such scenario emerges from initial condition(s) given by

$$
\begin{aligned}
\psi(x, 0) & =\sum_{j=-M}^{M} \psi_{S}(x-T-j d, 0 ;-\kappa, 0) \\
& +\sum_{j=-M}^{M} \psi_{S}(x+T-j d, 0 ; \kappa, \theta),
\end{aligned}
$$

where the first sum relates to sublattice B, and the second to sublattice A. The parameter $d$ defines the size of the unit cell, and $T$ is the initial offset between the two sublattices. The next-nearest-neighbor (NNN) tunneling in our SSH lattice is negligible, $t^{\prime \prime} \approx 0$. Due to the presence of nonlinearity, soliton interaction results in a dynamically evolving optically-induced lattice. To study its properties, we study the eigenvalues $\beta_{N L, n}(z)$ and the eigenmodes $\phi_{N L, n}(x, z)$ of the (nonlinearly) optically induced lattice potential $V(x, z)=-\gamma|\psi(x, z)|^{2}$, defined by $H \phi_{N L, n}=\beta_{N L, n} \phi_{N L, n}$; here $H=-(2 k)^{-1} \partial_{x x}+V$. An equivalent approach for evolving nonlinear topological lattices was adopted in Ref. [16].

In Fig. 2(a) we show the numerically calculated intensity of the evolving soliton lattice. The two sublattices propagate in opposite directions and periodically collide, but they maintain their sublattice structures and prop- 

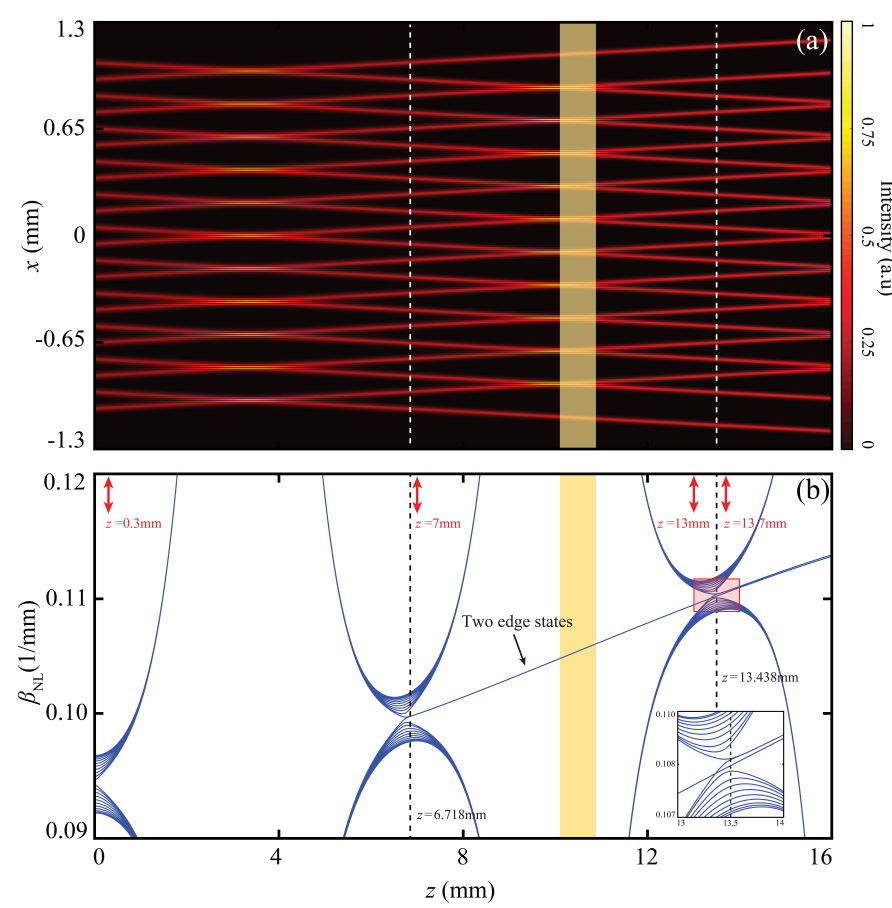

FIG. 2: Intensity (a) and spectrum (b) of the SSH soliton lattice evolving with propagation distance $z$. Locations where topological phase transitions happen are indicated with vertical dashed lines, while crossover region is highlighted by the yellow stripe. Topological phase transitions occur at the gap-closing points, after which two extended eigenmodes are pulled from the bands into the gap and become topological edge states (i.e., the phase transition here is from trivial-tonontrivial phase). Between these closing points, there is a crossover from nontrivial-to-trivial phase via decoupling of the edge states from the bulk of the lattice, which can be understood by comparing (a) with Fig. 1(d). At the second transition stage, two new edge states emerge, as seen clearly in the zoom-in inset in (b), while the old ones turn into decoupled walk-off solitons. Parameters: $M=5, T=d / 4=50 \mu \mathrm{m}$, $\theta=\pi, x_{0}=18.0 \mu \mathrm{m}, \kappa=5, k=1.71 \times 10^{7} \mathrm{~m}^{-1}$, and $\gamma I_{0}=\left(k x_{0}^{2}\right)^{-1}$. See text for the explanation of selected $z$ distances.

agation directions intact after every collision, which is ensured by the colliding properties of (Kerr-type) solitons [31. The intercell and intracell distances are equal at $z=0$, because we have chosen $T=d / 4 ; \kappa>0 \mathrm{im}$ plies that the sublattices initially approach each other. Thus, in the $z$-interval from $z=0$ until the first collision, the soliton lattice has the structure of the trivial SSH lattice, see Fig. 2(a). After the first collision, the lattice retains its trivial topology until the intercell and intracell distances became equal again for the first time after $z=0$. This point is denoted with a vertical dashed line at $z=6.718 \mathrm{~mm}$ in Fig. 2. At that point, the lattice undergoes a topological phase transition from the trivial to the nontrivial SSH soliton lattice, illustrated in real space in Figs. 2(a) and 1(c-left).
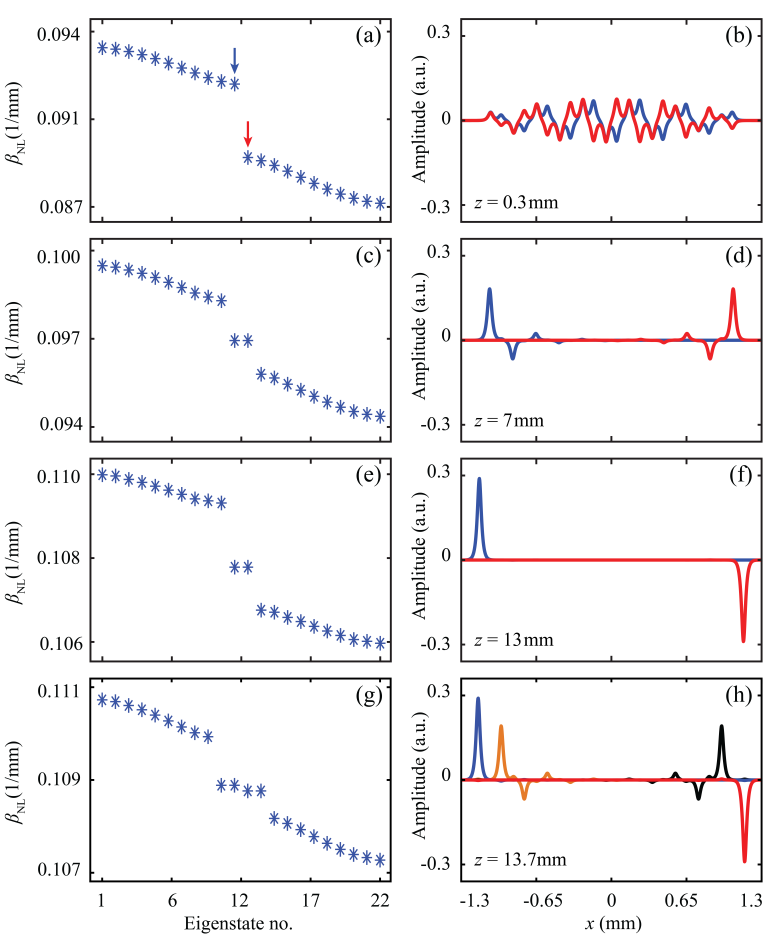

FIG. 3: Spectra of the evolving soliton lattice (left column) and selected eigenmodes $\phi_{N L, n}$ (right column) at propagation distances indicated by red arrows in Fig. 2 (a) Spectrum and (b) two eigenmodes in the trivial phase at $z=0.3 \mathrm{~mm}$. The two eigenmodes are closest to the gap as indicated with arrows in (a). (c) Spectrum and (d) topological localized states in the nontrivial phase at $z=7 \mathrm{~mm}$, just after the first topological phase transition. (e) Spectrum and (f) localized states after the crossover from the nontrivial to the trivial phase at $z=13 \mathrm{~mm}$. The states are localized solely in the outermost solitons, their amplitude is zero in the bulk of the soliton lattice, which is in contrast from the amplitude-phase structure of topological edge states shown in (d). (g) Spectrum and (h) localized states at $z=13.7 \mathrm{~mm}$, after the 2nd phase transition. Two of the localized states are topological (black and orange lines), whereas the other two are outermost solitons (blue and red lines).

An ultimate signature of the dynamical topological phase transition is illustrated in Fig. 2(b), which shows the band-gap structure of the evolving soliton lattice. We see that for $z$ values up to the first topological phase transition point at $z=6.718 \mathrm{~mm}$, there are two bands without any states in the gap. At the transition point the gap closes and immediately re-opens, where two eigenvalues are pulled from the bands to stay within the gap. These isolated eigenvalues correspond to the topologically nontrivial edge states of the SSH soliton lattice, with characteristic phase and amplitude structure, illustrated in Fig. 3(d) [16, 30, 34. They dynamically emerged at the transition point. Gap closing is an inevitable and necessary ingredient of a topological phase transition that is clearly illustrated in Fig. 2(b).

In order to fully unveil the behavior of our system, 
we explore the band gap structure and the modes of the SSH soliton lattice before the transition in Fig. 3(a,b) (for concreteness we consider $z=0.3 \mathrm{~mm}$ ), and just after the transition in Fig. 3(c,d) (at $z=7 \mathrm{~mm}$ ). At $z=0.3 \mathrm{~mm}$ there are two bands separated by the gap, see Fig. 3(a). All eigenmodes of the lattice are extended. In Fig. 3(b) we plot the two extended modes with eigenvalues closest to the gap. At the phase transition, these two extended modes are pulled from the band into the gap, see Fig. 3(c); at this point they became localized topological edge modes of the $\mathrm{SSH}$ soliton lattice, illustrated in Fig. 3(d). We see that both of them have the characteristic features of the topological edge modes: their amplitude is nonzero only in odd lattice sites (counting from the edge inwards), and the neighboring peaks in the mode amplitude are out of phase (see e.g. [16, 30, 34]).

A glance at Fig. 2(b) shows an interesting feature of the evolving spectrum at $z=13.438 \mathrm{~mm}$ : another gap closing and re-opening occurs, where two eigenstates bifurcate from the bands to become localized in the gap; see the inset in Fig. 2(b) and Fig. 3(g) and (h). This appears to be another topological phase transition from the trivial to the nontrivial SSH lattice. However, if this interpretation is correct (as we show in what follows), it means that the system is converted from the nontrivial to the trivial regime in between the two gap closing points shown in Fig. 2(b). This conversion is not a topological phase transition because the gap remains open at all propagation distances between $6.718 \mathrm{~mm}$ and $13.438 \mathrm{~mm}$.

To explain this intriguing phenomenon, we need to resort to the real space dynamics in Fig. 2(a), and explore the region shaded in yellow where the soliton collisions take place. In this region two outermost solitons become separated from the lattice, because the distance to their nearest neighbors becomes $d$, which is the NNN distance in the SSH lattice, and thus the tunneling probability from these outermost solitons to the bulk of the SSH lattice is practically zero. The eigenvalues corresponding to the outermost solitons are in the gap [see Fig. 3(e)], so the eigenmodes are obviously localized [see Fig. 33(f)], but their amplitude-phase structure does not possess the feature of topological edge states illustrated in Fig. 3(d). Thus, in the yellow region, two outermost solitons are actually decoupled from the SSH lattice, which leads to the crossover from the topologically nontrivial to the trivial phase. This crossover is fully equivalent to a gradual process of pulling two outermost lattice sites of the nontrivial SSH lattice into infinity, as illustrated in Fig. 11(d).

The existence of the crossover is in full agreement with the observation and interpretation of the gap closing point at $z=13.438 \mathrm{~mm}$ in Fig. 2(b) described above. This pattern of alternating sequence of events - dynamical topological phase transitions (trivial-to-nontrivial phase $\rightarrow$ crossover via decoupling of the outermost solitons (nontrivial-to-trivial phase), repeats itself during propagation until the two sublattices become separated and evolve without further collisions. The duration of this sequence depends on the number of solitons in each sublattice. The sublattice constant $d$ is chosen sufficiently large so that the NNN tunneling probability is negligible; therefore, when sublattices become separated, we can regard this system as a set of independent solitons.

In conclusion, we have found dynamical topological phase transitions in evolving nonlinear SSH soliton lattices, which are classified as emergent nonlinear topological phenomena, because they cease to exist if nonlinearity is turned off. These phase transitions convert the SSH soliton lattices from the topologically trivial-to-nontrivial phase, and are evinced by the gap closing and re-opening accompanied by emergence of two localized topological edge states. The eigenvalues of these edge states are pulled from the bands into the gap at the phase transition point. In addition, we have found a crossover from the topologically nontrivial-to-trivial regimes, which occurs via decoupling of the nontrivial edge states from the bulk of the lattice. These two opposing events occur one after the other in succession during the nonlinear dynamical evolution of the system. We have used the widely present Kerr-type nonlinearity to demonstrate our findings; it should be mentioned that in nonlinear saturable media such as photorefractive crystals, soliton collisions are typically not elastic (e.g., there can be fission and fusion of solitons), which means that direct observation of topological phase transitions proposed here should be more challenging (albeit not impossible) in saturable media. Nevertheless, we envisage this work will lead to exciting directions of fundamental research in nonlinear topological photonics.

This research is supported in part by the QuantiXLie Center of Excellence, a project co-financed by the Croatian Government and European Union through the European Regional Development Fund - the Competitiveness and Cohesion Operational Programme (Grant KK.01.1.1.01.0004), the National Key R\&D Program of China under Grant No. 2017YFA0303800, the National Natural Science Foundation (11922408, 91750204, 11674180), PCSIRT, and the 111 Project (No. B07013) in China. R.M. acknowledges support from the NSERC Discovery and the Canada Research Chair Programs. R.M. is affiliated to 5 as an adjoint faculty. D.B. acknowledges support from the 66 Postdoctoral Science Grant of China. We acknowledge support from the CroatianChinese Scientific \& Technological Cooperation.

* Electronic address: zgchen@nankai.edu.cn

$\dagger$ Electronic address: hbuljan@phy.hr

[1] L. Lu, J.D. Joannopoulos, M. Soljačić, Nat. Photon. 8, 821-829 (2014).

[2] T. Ozawa, H. M. Price, A. Amo, N. Goldman, M. Hafezi, 
L. Lu, M. C. Rechtsman, D. Schuster, J. Simon, O. Zilberberg, I. Carusotto, Rev. Mod. Phys. 91, 015006 (2019).

[3] F.D.M. Haldane, S. Raghu, Phys. Rev. Lett. 100, 013904 (2008); S. Raghu, F.D.M. Haldane, Phys. Rev. A 78, 033834 (2008).

[4] Z. Wang, Y. Chong, J. D. Joannopoulos, M. Soljačić, Nature 461, 772 (2009).

[5] Y. Lumer, Y. Plotnik, M. C. Rechtsman, M. Segev, Phys. Rev. Lett. 111, 243905 (2013).

[6] M.J. Ablowitz, C.W. Curtis, and Y-P. Ma, Phys. Rev. A 90, 023813 (2014).

[7] D. Leykam, Y.D. Chong, Phys. Rev. Lett. 117, 143901 (2016).

[8] N. Malkova, I. Hromada, X. Wang, G. Bryant, and Z. Chen, Phys. Rev. A 80, 043806 (2009).

[9] Y. Hadad, A. B. Khanikaev and A. Alu, Phys. Rev. B 93, 155112 (2016).

[10] X. Zhou, Y. Wang, D. Leykam and Y.D. Chong, New J. Phys. 19, 095002 (2017).

[11] L. J. Maczewsky et al., Science 370, 701 (2020).

[12] Y. T. Katan, R. Bekenstein, M. Bandres, Y. Lumer, Y. Plotnik, and M. Segev, in Conference on Lasers and Electro-Optics, OSA Technical Digest (Optical Society of America, 2016), paper FM3A.6.

[13] S. Mukherjee, and M. C. Rechtsman, Science 368, 856 (2020).

[14] D. D. Solnyshkov, O. Bleu, B. Teklu, G. Malpuech, Phys. Rev. Lett. 118, 023901 (2017).

[15] D. A. Dobrykh, A. V. Yulin, A. P. Slobozhanyuk, A. N. Poddubny, Y. S. Kivshar, Phys. Rev. Lett. 121, 163901 (2018).

[16] S. Xia, D. Jukić, N. Wang, D. Smirnova, L. Smirnov, L. Tang, D. Song, A. Szameit, D. Leykam, J. Xu, Z. Chen, and H. Buljan, Light Sci. Appl. 9(1), 147 (2020).

[17] A. Bisianov, M. Wimmer, U. Peschel, O. A. Egorov, Phys. Rev. A 100, 063830 (2019).

[18] W. Zhang, X. Chen, Y.V. Kartashov, V.V. Konotop, and F. Ye, Phys. Rev. Lett. 123, 254103 (2019).

[19] S. Kruk, A. Poddubny, D. Smirnova, L. Wang, A. Slobozhanyuk, A. Shorokhov, I. Kravchenko, B. LutherDavies, and Y. Kivshar, Nat. Nanotechnol. 14, 126 (2019).

[20] Y. Wang, L.-J. Lang, C. H. Lee, B. Zhang, Y. D. Chong, Nat. Commun. 10, 1102 (2019).

[21] D. Smirnova, D. Leykam, Y. Chong, Y. Kivshar, Appl. Phys. Rev. 7, 021306 (2020).

[22] M.A. Bandres, S, Wittek, G. Harari, M. Parto, J. Ren, M. Segev, D.N. Christodoulides, M. Khajavikhan, Science 359, eaar4005 (2018).

[23] G. Harari, M.A. Bandres, Y. Lumer, M.C. Rechtsman, Y.D. Chong, M. Khajavikhan, D.N. Christodoulides, M. Segev, Science 359, eaar4003 (2018)

[24] P. St-Jean, V. Goblot, E. Galopin, A. Lemaître, T. Ozawa, L. Le Gratiet, I. Sagnes, J. Bloch, and A. Amo, Nat. Photon. 11, 651-656 (2017).
[25] B. Bahari, A. Ndao, F. Vallini, A. El Amili, Y. Fainman, B Kanté, Science 358, 636 (2017).

[26] H. Zhao, P. Miao, M.H. Teimourpour, S. Malzard, R. ElGanainy, H. Schomerus, and L. Feng Nat. Commun. 9, 981 (2018).

[27] M. Parto, S. Wittek, H. Hodaei, G. Harari, M.A. Bandres, J. Ren, M.C. Rechtsman, M. Segev, D.N. Christodoulides, and M. Khajavikhan, Phys. Rev. Lett. 120, 113901 (2018).

[28] S. Xia, D. Kaltsas, D. Song, I. Komis, J. Xu, A. Szameit, H. Buljan, K.G. Makris, Z. Chen, https://arxiv.org/abs/2010.16294

[29] N. Pernet, P. St-Jean, D. D. Solnyshkov, G. Malpuech, N. Carlon Zambon, B. Real, O. Jamadi, A. Lemaître, M. Morassi, L. Le Gratiet, T. Baptiste, A. Harouri, I. Sagnes, A. Amo, S. Ravets, J. Bloch, https://arxiv.org/abs/2101.01038

[30] W.P. Su, J.R. Schrieffer, A.J. Heeger, Phys. Rev. Lett. 42, 1698 (1979).

[31] Z. Chen, M. Segev, and D. N. Christodoulides, Rep. Prog. Phys. 75, 086401 (2012).

[32] J. Zak, Phys. Rev. Lett. 62, 2747-2750 (1989).

[33] Y. Kraus, Y. Lahini, Z. Ringel, M. Verbin, and O. Zilberberg Phys. Rev. Lett. 109106402 (2012).

[34] N. Malkova, I. Hromada, X. Wang, G. Bryant, Z. Chen, Opt. Lett. 34, 1633-1635 (2009).

[35] R. Keil, J. M. Zeuner, F. Dreisow, M. Heinrich, A. T'unnermann, S. Nolte, and A. Szameit, Nat. Commun. 4, 1368 (2013).

[36] M. Xiao, Z.Q. Zhang, C.T. Chan, Phys. Rev. X 4, 021017 (2014).

[37] A. Blanco-Redondo, I. Andonegui, M. J. Collins, G. Harari, Y. Lumer, M. C. Rechtsman, B. J. Eggleton, and M. Segev Phys. Rev. Lett. 116, 163901 (2016).

[38] S. Weimann, M. Kremer, Y. Plotnik, Y. Lumer, S. Nolte, K. G. Makris, M. Segev, M. C. Rechtsman, A. Szameit, Nat. Mater. 16, 433-438 (2017).

[39] A. Poddubny, A., Miroshnichenko, A., Slobozhanyuk, Y. Kivshar, ACS Photonics 1, 101-105 (2014).

[40] F. Bleckmann, Z. Cherpakova, S. Linden, A. Alberti, Phys. Rev. B 96, 045417 (2017).

[41] T. Kitagawa, M. A. Broome, A. Fedrizzi, M. S. Rudner, E. Berg, I. Kassal, A. Aspuru-Guzik, E. Demler, and A. G. White, Nat. Commun. 3, 882 (2012).

[42] F. Cardano, A. D'Errico, A. Dauphin, M. Maffei, B. Piccirillo, C. de Lisio, G. De Filippis, V. Cataudella, E. Santamato, L. Marrucci, M. Lewenstein, and P. Massignan, Nat. Commun. 8, 15516 (2017).

[43] A. Blanco-Redondo, B. Bell, D. Oren, B.J. Eggleton, M. Segev, Science 362, 568 (2018).

[44] M. Bello, G. Platero, J.I. Cirac, A. González-Tudela, Sci. Adv. 5, eaaw0297 (2019).

[45] G. Agrawal, Nonlinear Fiber Optics, (Academic Press, Cambridge, 2013). 